Pacific Journal of Mathematic 


\section{ORTHOGONAL GROUPS OF POSITIVE DEFINITE MULTILINEAR FUNCTIONALS}

\section{Stephen Pierce}

Let $V$ be a finite dimensional vector space over the real numbers $R$ and let $T: V \rightarrow V$ be a linear transformation. If $\varphi: \times_{1}^{m} V \rightarrow R$ is a real multilinear functional and

$$
\varphi\left(T x_{1}, \cdots, T x_{m}\right)=\varphi\left(x_{1}, \cdots, x_{m}\right),
$$

$x_{1}, \cdots, x_{m} \in V, T$ is called an isometry with respect to $\varphi$. We say $\varphi$ is positive definite if $\varphi(x, \cdots, x)>0$ for all nonzero $x \in V$. In this paper we prove that if $\varphi$ is positive definite and $T$ is an isometry with respect to $\varphi$, then all eigenvalues of $T$ have modulus one and all elementary divisors of $T$ over the complex numbers are linear.

Let $V$ be an $n$-dimensional vector space over the real numbers $R$. Let $T: V \rightarrow V$ be a linear transformation of $V$. The following theorem $[1, \mathrm{Th} .3]$ is easy to prove:

THEOREM 1. There exists a positive definite symmetric quadratic form $\varphi: V \times V \rightarrow R$ such that

$$
\varphi(T x, T y)=\varphi(x, y), x, y \in V
$$

if and only if

1. all eigenvalues of $T$ have modulus 1 ;

2. all elementary divisors of $T$ over the complex numbers $C$ are linear.

Moreover, if $T$ satisfies (2), then there is a positive definite symmetric $\varphi$ such that (1) holds.

Theorem 1 can also be expressed in matrix theoretic terms. If $A$ is a real $n \times n$ positive definite symmetric matrix and $X$ is any automorph of $A$;

$$
X^{T} A X=A,
$$

then $X$ satisfies (2); moreover, if an $n \times n$ matrix $X$ satisfies (2), then there is a positive definite symmetric $A$ such that (3) holds.

Let $\varphi: \times{ }_{1}^{m} V \rightarrow R$ be a real multilinear functional. Let $H$ be a subgroup of the symmetric group $S_{m}$. If

$$
\varphi\left(x_{\sigma(1)}, \cdots, x_{\sigma(m)}\right)=\varphi\left(x_{1}, \cdots, x_{m}\right)
$$


for all $\sigma \in H$ and all $x_{i} \in V, i=1, \cdots, m$, then $\varphi$ is said to be symmetric with respect to $H$. If

$$
\varphi\left(T x_{1}, \cdots, T x_{m}\right)=\varphi\left(x_{1}, \cdots x_{m}\right)
$$

for all $x_{1}, \cdots, x_{m} \in V, T$ is called an isometry of $V$ with respect to $\varphi$. (Note that if $m>2$, (5) has no matrix analogue). Let $\Omega_{m}(H, T)$ be the set of all $\varphi$ satisfying (4) and (5). Clearly $\Omega_{m}(H, T)$ is a subspace of the vector space of all multilinear functionals symmetric with respect to $H$. We say $\varphi$ is positive definite if

$$
\varphi(x, \cdots, x)>0
$$

for all nonzero $x$ in $V$. The set of all positive definite $\varphi$ in $\Omega_{m}(H, T)$ is denoted by $P_{m}(H, T)$. It is clear that $P_{m}(H, T)$ is a (possibly empty) convex cone in $\Omega_{m}(H, T)$.

The following result [1] was proved as a partial generalization of Theorem 1.

TheOREm 2. Let $T: V \rightarrow V$ be linear. If $P_{m}(H, T)$ is nonempty, then

(a) $m$ is even

(b) every eigenvalue $\gamma$ of $T$ has modulus 1

(c) elementary divisors of $T$ corresponding to $\gamma= \pm 1$ are linear. Conversely, if $m$ is even, all eigenvalues of $T$ are \pm 1 , and all elementary divisors of $T$ are linear, then $P_{m}(H, T)$ is nonempty.

We conjectured that if $P_{m}(H, T)$ is nonempty, then $(c)$ can be replaced by $\left(c^{\prime}\right)$ "all elementary divisors of $T$ over the complex field are linear." This would provide a complete generalization of Theorem 2, and thus justify (6) as a definition of a positive definite multilinear functional. The purpose of this paper is to prove this conjecture.

Theorem 3. If $P_{m}(H, T)$ is nonempty, then

(a) $m$ is even

(b) all eigenvalues of $T$ have modulus 1

$\left(\mathrm{c}^{\prime}\right)$ all elementary divisors of $T$ over $C$ are linear. Conversely, if (a), (b), and $\left(\mathrm{c}^{\prime}\right)$ hold, then $P_{m}(H, T)$ is nonempty.

2. Proof of Theorem 3. Assume that $P_{m}(H, T)$ is nonempty. Parts (a) and (b) follow from Theorem 2. We now prove two lemmas.

Lemma 1. If $\gamma$ is an eigenvalue of $T$ and $(x-\gamma)^{k}, k>1$, is a nonlinear elementary divisor of $T$ corresponding to $\gamma$, then $\gamma^{m} \neq 1$ for any integer $m$. 
Proof. Since $T$ is a real transformation, it has a real elementary divisor

$$
[(x-\gamma)(x-\bar{\gamma})]^{k}
$$

(By Theorem 2, $\gamma$ cannot be real in this case.) Let $W$ be the invariant subspace of $T$ determined by (7), and let $S$ be the restriction of $T$ to $W$. Then $S$ is an isometry of $W$ with respect to $\varphi$, and hence $S^{r}$ is also an isometry for any integer $r$. Now if $\gamma^{r}=1$, then all eigenvalues of $S^{r}$ are 1, and hence Theorem 2 implies that all elementary divisors of $S^{r}$ are linear. Therefore, $S^{r}$ is the identity on $W$, and thus, the elementary divisors of $S$ are linear, a contradiction.

Lemma 2. If Theorem 3 is true for the case $H=S_{m}$, then it is true for any subgroup $H$ of $S_{m}$.

Proof. Let $H$ be a subgroup of $S_{m}$ and let $\varphi \in P_{m}(H, T)$. For each $\sigma \in S_{m}$, define

$$
\varphi_{\sigma}\left(x_{1}, \cdots, x_{m}\right)=\varphi\left(x_{\sigma(1)}, \cdots, x_{\sigma(m)}\right),
$$

$x_{1}, \cdots, x_{m} \in V$. In general, $\varphi_{o}$ is not symmetric with respect to $H$, but $\varphi_{\sigma}$ is positive definite and $T$ is an isometry with respect to $\varphi_{\sigma}$. Set

$$
\psi=\sum_{\sigma \in S_{m}} \varphi_{\sigma}
$$

Clearly $\psi$ is positive definite, and $T$ is an isometry with respect to $\psi$. Moreover, for any $\tau \in S_{m}$, and $x_{1}, \cdots, x_{m} \in V$,

$$
\begin{aligned}
\psi\left(x_{\tau(1)}, \cdots, x_{\tau(m)}\right) & =\sum_{\sigma \in S_{m}} \varphi_{\sigma}\left(x_{\tau(1)}, \cdots, x_{\tau(m)}\right) \\
& =\sum_{\sigma \in S_{m}} \varphi\left(x_{\tau \sigma(1)}, \cdots, x_{\tau \sigma(m)}\right) \\
& =\sum_{\mu \in S_{m}} \varphi\left(x_{\mu(1)}, \cdots, x_{\mu(m)}\right) \\
& =\sum_{\mu \in S_{m}} \varphi_{\mu}\left(x_{1}, \cdots, x_{m}\right) \\
& =\psi\left(x_{1}, \cdots, x_{m}\right) .
\end{aligned}
$$

Thus $i \in P_{m}\left(S_{m}, T\right)$, and hence the elementary divisors of $T$ are linear. This proves Lemma 2.

We may assume henceforth that $H=S_{m}$ and abbreviate $P_{m}\left(S_{m}, T\right)$ to $P_{m}$. If $P_{m}$ is nonempty, and $T$ has a nonlinear elementary divisor over $C$ corresponding to the eigenvalue $\gamma=a+i b(b \neq 0)$, then there exist four linearly independent vectors $v_{1}, \cdots, v_{4}$ in $V$ such that 


$$
\begin{aligned}
& T v_{1}=a v_{1}-b v_{2} \\
& T v_{2}=b v_{1}+a v_{2} \\
& T x_{3}=v_{2}+a v_{3}-b v_{4} \\
& T v_{4}=b v_{3}+a v_{4} .
\end{aligned}
$$

Let $\bar{V}$ be the extension of $V$ to an $n$-dimensional space over $C$, i.e., $\bar{V}$ consists of all vectors of the form $x+i y, x, y \in V$. By linear extension, we regard $T$ as a linear transformation of $\bar{V}$, and by multilinear extension, $\varphi$ becomes a complex valued multilinear functional on $\times_{1}^{m} \bar{V}$. Equation (5) still holds in $\bar{V}$, but $\varphi$ is no longer positive definite. Set

$$
\begin{aligned}
& e_{1}=v_{1}+i v_{2}, e_{2}=v_{1}-i v_{2} \\
& e_{3}=v_{3}+i v_{4}, e_{4}=v_{3}-i v_{4}
\end{aligned} .
$$

From (10) and (11),

$$
\begin{gathered}
T e_{1}=\gamma e_{1}, \quad T e_{2}=\bar{\gamma} e_{2} \\
T e_{3}=\gamma e_{3}+v_{2}, T e_{4}=\bar{\gamma} e_{4}+v_{2} .
\end{gathered}
$$

By Lemma 1, $\gamma$ is not a root of unity; thus,

$$
\begin{aligned}
\varphi\left(e_{1}, \cdots, e_{1}, e_{2}, \cdots e_{2}\right) & =\varphi\left(T e_{1}, \cdots, T e_{1}, T e_{2}, \cdots, T e_{2}\right) \\
& =\gamma^{k} \bar{\gamma}^{m-k} \varphi\left(e_{1}, \cdots e_{1}, e_{2}, \cdots, e_{2}\right) \\
& =0
\end{aligned}
$$

unless $k=m-k$, where $k$ is the number of times $e_{1}$ occurs in (13). With $r=m / 2$, we set

$$
\varphi\left(e_{1}, \stackrel{r}{\cdots}, e_{1}, e_{2}, \stackrel{r}{\cdots}, e_{2}\right)=\nu .
$$

Now $\nu \neq 0$; otherwise

$$
\begin{aligned}
\varphi\left(v_{1}, \cdots, v_{1}\right) & =2^{-m} \varphi\left(e_{1}+e_{2}, \cdots, e_{1}+e_{2}\right) \\
& =0
\end{aligned}
$$

contradicting (6). (Note that we are using the assumption that $\varphi$ is symmetric with respect to $S_{m}$; this gives us a convenient way of sorting expressions such as those on the right side of (14).)

Let $\mu=\varphi\left(v_{1}, \cdots, v_{1}, e_{3}\right)$. Using (13) and (14), we compute,

$$
\begin{aligned}
\mu & =2^{-m+1} \varphi\left(e_{1}+e_{2}, \cdots, e_{1}+e_{2}, e_{3}\right) \\
& =2^{-m+1} \varphi\left(\gamma e_{1}+\bar{\gamma} e_{2}, \cdots, \gamma e_{1}+\bar{\gamma} e_{2}, \gamma e_{3}+v_{2}\right) \\
& =2^{-m+1} \varphi\left(\gamma e_{1}+\bar{\gamma} e_{2}, \cdots \gamma e_{1}+\bar{\gamma} e_{2}, \gamma e_{3}+\frac{e_{1}-e_{2}}{2 i}\right) \\
& =-2^{-m} i\left(\begin{array}{c}
m-1 \\
r
\end{array}\right)(\bar{\gamma}-\gamma) \nu+\gamma 2^{-m+1} \varphi\left(\gamma e_{1}+\bar{\gamma} e_{2}, \cdots \gamma e_{1}+\bar{\gamma} e_{2}, e_{3}\right)
\end{aligned}
$$




$$
\begin{aligned}
& \begin{aligned}
=-2^{-m} i\left(\begin{array}{c}
m-1 \\
r
\end{array}\right)(\bar{\gamma}-\gamma) \nu+\gamma 2^{-m+1} \\
\\
\varphi\left(\gamma^{2} e_{1}+\bar{\gamma}^{2} e_{2}, \cdots, \gamma^{2} e_{1}+\bar{\gamma}^{2} e_{2}, \gamma e_{3}+\frac{e_{1}-e_{2}}{2 i}\right) \\
=-2^{-m} i\left(\begin{array}{c}
m-1 \\
r
\end{array}\right)\left(2 \bar{\gamma}-\gamma-\gamma^{3}\right) \nu+\gamma^{2} 2^{-m+1} \\
\varphi\left(\gamma^{2} e_{1}+\bar{\gamma}^{2} e_{2}, \cdots, \gamma^{2} e_{1}+\bar{\gamma}^{2} e_{2}, e_{3}\right) .
\end{aligned}
\end{aligned}
$$

Continuing this procedure, we obtain for any positive integer $s$

$$
\begin{aligned}
\mu=-2^{-m} i\left(\begin{array}{c}
m-1 \\
r
\end{array}\right)\left(s \bar{\gamma}-\sum_{j=0}^{s-1} \gamma^{2 j+1}\right) \nu+\gamma^{s} 2^{-m+1} \\
\varphi\left(\gamma^{s} e_{1}+\bar{\gamma}^{s} e_{2}, \cdots \gamma^{s} e_{1}+\bar{\gamma}^{s} e_{2}, e_{3}\right) .
\end{aligned}
$$

Let

$$
f(z)=z \varphi\left(z e_{1}+\bar{z} e_{2}, \cdots, z e_{1}+\bar{z} e_{2}, e_{3}\right),
$$

where $z$ is a complex variable. Then $f$ is a continuous function of $z$ on the complex plane, and hence $f$ is bounded on the unit circle. Moreover, since $\gamma$ is not a root of unity (in particular, $\gamma \neq \pm 1$ ),

$$
\sum_{j=0}^{s-1} \gamma^{2 j-1}
$$

is also bounded as $s$ becomes large. Thus, letting $s$ approach infinity in (15) forces $\mu$ to become infinite, a contradiction. This proves Theorem 3 in one direction.

Now suppose all eigenvalues of $T$ are 1 in absolute value and all elementary divisors of $T$ are linear over $C$. Let 1 ( $p$ times), -1 ( $q$ times) and $\gamma_{j}, \bar{\gamma}_{j}=a_{j} \pm i b_{j},\left|\gamma_{j}\right|=1, j=1, \cdots, t$, be the eigenvalues of $T$. Then there is a basis of $V, v_{1}, \cdots, v_{p}, u_{1}, \cdots, u_{q}, x_{1}, y_{1}, \cdots x_{t}, y_{t}$ such that

$$
\begin{aligned}
& T v_{j}=v_{j}, j=1, \cdots, p \\
& T u_{j}=-u_{j}, j=1, \cdots, q \\
& T x_{j}=a_{j} x_{j}-b_{j} y_{j}, j=1, \cdots, t \\
& T y_{j}=b_{j} x_{j}+a_{j} y_{j}, j=1, \cdots, t .
\end{aligned}
$$

Set

$$
\begin{aligned}
& w_{j}=x_{j}+i y_{j} \\
& \bar{w}_{j}=x_{j}-i y_{j}, j=1, \cdots, t .
\end{aligned}
$$

Then $v_{1}, \cdots, v_{p}, u_{1}, \cdots, u_{q}, w_{1}, \bar{w}_{1}, \cdots, w_{t}, \bar{w}_{t}$ form a basis of $\bar{V}$ of eigenvectors of $T$. Let $f_{1}, \cdots, f_{p}, g_{1}, \cdots, g_{q}, h_{1}, k_{1}, \cdots, h_{t}, k_{t}$ be the corresponding dual basis. If $l_{1}, \cdots, l_{m}$ are linear functionals on a space $V$, then $l_{1} \cdots l_{m}$ is the $m$-linear functional on $\times_{1}^{m} V$ such that 


$$
l_{1} \cdots l_{m}\left(x_{1}, \cdots, x_{m}\right)=\prod_{i=1}^{m} l_{i}\left(x_{i}\right)
$$

Define $\varphi$ as follows:

$$
\varphi=\sum_{j=1}^{p} f_{j}^{m}+\sum_{j=1}^{q} g_{j}^{m}+\sum_{j=1}^{t}\left[\left(h_{j} k_{j}\right)^{r}+\left(\bar{h}_{j} \bar{k}_{j}\right)^{r}\right],
$$

where $r=m / 2$ and $\bar{f}(v)=\overline{f(v)}$. Now $\bar{h}_{j}$ and $\bar{k}_{j}$ are not linear on the complex space $\bar{V}$, but they are complex valued linear functionals on $V$, i.e., they are linear functionals on $V$ but are not in the dual space of $V$. Thus $\varphi$ is a real multilinear functional on $V$. Set

$$
\psi=\sum_{\sigma \in S_{m}} \varphi_{\sigma}
$$

We assert that $\psi \in P_{m}(H, T)$. Clearly $\psi$ is symmetric with respect to $S_{m}$, and thus with respect to any subgroup $H$ of $S_{m}$. It remains to show that $\psi$ is positive definite and that $T$ is an isometry with respect to $\psi$. It suffices to prove these last two properties for $\varphi$. Let

$$
x=\sum_{j=1}^{p} \alpha_{j} v_{j}+\sum_{j=1}^{q} \beta_{j} u_{j}+\sum_{j=1}^{t}\left(\delta_{j} x_{j}+\lambda_{j} y_{j}\right)
$$

be an arbitrary vector of $V$. Then from (17),

$$
\varphi(x, \cdots, x)=\sum_{j=1}^{p} \alpha_{j}^{n}+\sum^{q} \beta_{j}^{m}+2 \sum_{j=1}^{t}\left[\left(\frac{\delta_{j}}{2}\right)^{2}+\left(\frac{\lambda_{j}}{2}\right)^{2}\right]^{r} .
$$

Since $m$ is even and $\alpha_{j}, \beta_{j}, \delta_{j}, \lambda_{j}$ are all real, $\varphi$ is positive definite. Now let $z_{k}, k=1, \cdots, m$, be arbitrary vectors in $V$, with

$$
z_{k}=\sum_{j=1}^{p} a_{k j} v_{j}+\sum_{j=1}^{q} b_{k j} u_{j}+\sum_{j=1}^{t}\left(c_{k j} x_{j}+d_{k j} y_{j}\right) .
$$

Then

$$
\begin{aligned}
\varphi\left(z_{1}, \cdots, z_{m}\right)= & \sum_{j=1}^{p} \prod_{k=1}^{m} a_{k j}+\sum_{j=1}^{q} \prod_{k=1}^{m} b_{k j} \\
& +\sum_{j=1}^{t} \prod_{k=1}^{r}\left(\frac{c_{2 k-1, j}}{2}+\frac{d_{2 k-1, j}}{2 i}\right)\left(\frac{c_{2 k, j}}{2}-\frac{d_{2 k, j}}{2 i}\right) \\
& +\sum_{j=1}^{t} \prod_{k=1}^{r}\left(\frac{c_{2 k-1, j}}{2}-\frac{d_{2 k-1, j}}{2 i}\right)\left(\frac{c_{2 k, j}}{2}+\frac{d_{2 k, j}}{2 i}\right) .
\end{aligned}
$$

From (16)

$$
\begin{aligned}
T z_{k}= & \sum_{j=1}^{p} a_{k j} v_{j}+\sum_{j=1}^{q}\left(-b_{k j}\right) u_{j} \\
& +\sum_{j=1}^{t}\left(a_{j} c_{k j}+b_{j} d_{k j}\right) x_{j}+\left(a_{j} d_{k j}-b_{j} c_{k j}\right) y_{j},
\end{aligned}
$$


$k=1, \cdots, m$. Let

$$
\begin{gathered}
e_{k j}=a_{j} c_{k j}+b_{j} d_{k j} \\
f_{k j}=a_{j} d_{k j}-b_{j} c_{k j} .
\end{gathered}
$$

Then from (19) and (20)

$$
\begin{aligned}
\varphi\left(T z_{1}, \cdots, T z_{m}\right)= & \sum_{j=1}^{p} \prod_{k=1}^{m} a_{k j}+\sum_{j=1}^{q} \prod_{k=1}^{m}\left(-b_{k j}\right) \\
& +\sum_{j=1}^{t} \prod_{k=1}^{m}\left(\frac{e_{2 k-1, j}}{2}+\frac{f_{2 k-1, j}}{2 i}\right)\left(\frac{e_{2 k, j}}{2}-\frac{f_{2 k, j}}{2 i}\right) \\
& +\sum_{j=1}^{t} \prod_{k=1}^{m}\left(\frac{e_{2 k-1, j}}{2}-\frac{f_{2 k-1, j}}{2}\right)\left(\frac{e_{2 k, j}}{2}+\frac{f_{2 k, j}}{2 i}\right) .
\end{aligned}
$$

It is easily verified that

$$
\begin{aligned}
& \frac{e_{k j}}{2}+\frac{f_{k j}}{2 i}=\bar{\gamma}_{j}\left(\frac{c_{k j}}{2}+\frac{d_{k j}}{2 i}\right) \\
& \frac{e_{k j}}{2}-\frac{f_{k j}}{2 i}=\gamma_{j}\left(\frac{c_{k j}}{2}-\frac{d_{k j}}{2 i}\right) .
\end{aligned}
$$

Using (22) in (21) and the fact that $\left|\gamma_{j}\right|=1$, we obtain

$$
\varphi\left(T z_{1}, \cdots, T z_{m}\right)=\varphi\left(z_{1}, \cdots, z_{m}\right) \text {. }
$$

This completes the proof of Theorem 3 .

\section{REFERENCES}

1. M. Marcus and S. Pierce, Positive definite multilinear functionals, Pacific J. Math. (to appear)

2. S. Perlis, Theory of matrices, Addison-Wesley, 1958.

Received July 8, 1960. This work was done while the author was a National Academy of Sciences-National Research Council Postdoctoral Research Associate at the National Bureau of Standards, Washington, D. C., 1968-70.

National Bureau of Standards

WAShington, D. C. 



\section{PACIFIC JOURNAL OF MATHEMATICS}

\section{EDITORS}

H. SAMELSON

Stanford University

Stanford, California 94305

\section{RichaRd PIERCe}

University of Washington

Seattle, Washington 98105
J. DUGUNDJI

Department of Mathematics

University of Southern California

Los Angeles, California 90007

RICHARD ARENS

University of California

Los Angeles, California 90024

\section{ASSOCIATE EDITORS}

E. F. BECKENBACH

B. H. NeUManN

F. WOLF

K. YosHIDA

\section{SUPPORTING INSTITUTIONS}

UNIVERSITY OF BRITISH COLUMBIA CALIFORNIA INSTITUTE OF TECHNOLOGY UNIVERSITY OF CALIFORNIA MONTANA STATE UNIVERSITY

UNIVERSITY OF NEVADA

NEW MEXICO STATE UNIVERSITY

OREGON STATE UNIVERSITY

UNIVERSITY OF OREGON

OSAKA UNIVERSITY

UNIVERSITY OF SOUTHERN CALIFORNIA
STANFORD UNIVERSITY

UNIVERSITY OF TOKYO

UNIVERSITY OF UTAH

WASHINGTON STATE UNIVERSITY

UNIVERSITY OF WASHINGTON

${ }^{*} \quad{ }^{*} \quad{ }^{*}$
AMERICAN MATHEMATICAL SOCIETY
CHEVRON RESEARCH CORPORATION
TRW SYSTEMS
NAVAL WEAPONS CENTER




\section{Pacific Journal of Mathematics}

\section{Vol. 33, No. $1 \quad$ March, 1970}

Mir Maswood Ali, On some extremal simplexes ................... 1

Silvio Aurora, On normed rings with monotone multiplication........... 15

Silvio Aurora, Normed fields which extend normed rings of integers....... 21

John Kelly Beem, Indefinite Minkowski spaces..................... 29

T. F. Bridgland, Trajectory integrals of set valued functions ........... 43

Robert Jay Buck, A generalized Hausdorff dimension for functions and sets ......................................... 69

Vlastimil B. Dlab, A characterization of perfect rings . . . . . . . . . . . . 79

Edward Richard Fadell, Some examples in fixed point theory ............ 89

Michael Benton Freeman, Tangential Cauchy-Riemann equations and uniform approximation ............................. 101

Barry J. Gardner, Torsion classes and pure subgroups ................ 109

Vinod B. Goyal, Bounds for the solution of a certain class of nonlinear

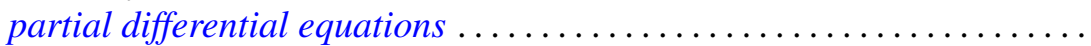

Fu Cheng Hsiang, On C, 1 summability factors of Fourier series at a given

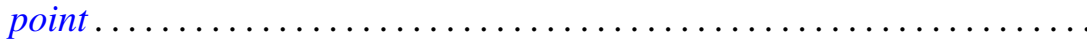

Lawrence Stanislaus Husch, Jr., Homotopy groups of PL-embedding

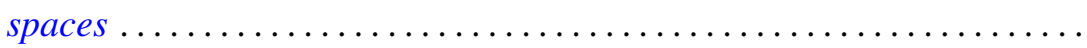

Daniel Ralph Lewis, Integration with respect to vector measures..........

Marion-Josephine Lim, $\mathscr{L}-2$ subspaces of Grassmann product spaces

Stephen J. Pierce, Orthogonal groups of positive definite multilinear functionals

W. J. Pugh and S. M. Shah, On the growth of entire functions of bounded index.

Siddani Bhaskara Rao and Ayyagari Ramachandra Rao, Existence of triconnected graphs with prescribed degrees . . .

Ralph Tyrrell Rockafellar, On the maximal monotonicity of subdifferential

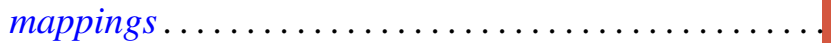

R. Shantaram, Convergence of a sequence of transformations of distribution functions. II ...............................

Julianne Souchek, Rings of analytic functions..............

Ted Joe Suffridge, The principle of subordination applied to functions of several variables...

Wei-lung Ting, On secondary characteristic classes in cobordism

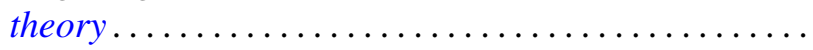

Pak-Ken Wong, Continuous complementors on $B^{*}$-algebras ...

Miyuki Yamada, On a regular semigroup in which the idempotents form a band. 